\title{
FIXED POINT THEOREMS ON GENERALIZED RECTANGULAR METRIC SPACES
}

\author{
O. K. ADEWALE ${ }^{1}$, J. O. OLALERU ${ }^{2}$, H. OLAOLUWA ${ }^{3}$ \\ and $H$. AKEWE ${ }^{4}$ \\ Department of Mathematics \\ University of Lagos \\ Lagos \\ Nigeria \\ e-mail: adewalekayode2@yahoo.com \\ jolaleru@unilag.edu.ng \\ holaoluwa@unilag.edu.ng \\ hakewe@unilag.edu.ng
}

\begin{abstract}
In this paper, we introduce the notion of generalized rectangular metric spaces which extends rectangular metric spaces introduced by Branciari. Analogues of the some well-known fixed point theorems are proved in this space. With an example, it is shown that a generalized rectangular metric space is neither a $G$-metric space nor a rectangular metric space. Our results generalize many known results in fixed point theory.
\end{abstract}

2020 Mathematics Subject Classification: 37C25, 47H10, 54H25, 55M20.

Keywords and phrases: rectangular metric spaces, $G$-metric spaces, fixed point.

Received February 22, 2021; Revised May 4, 2021

(C) 2021 Scientific Advances Publishers

This work is licensed under the Creative Commons Attribution International License (CC BY 3.0).

http://creativecommons.org/licenses/by/3.0/deed.en_US

Open Access (cc) (1)




\section{Introduction}

The notion of a distance between two points as the case may be is as old as human civilization. Frechet [8] put this notion into an abstract form and came out with metric spaces in early 19th century. Metric space is a crucial device in functional analysis, non-linear analysis and topology. This aspect of functional analysis has attracted the attention of many mathematicians due to the development of fixed point theory in it.

Many authors made attempt to generalize the usual notion of metric space $(X, d)$ to extend the known metric space theorems in a more general setting (see [1]-[12]). In attempt to generalize these usual metric spaces, 2-metric spaces, $D$-metric spaces, $G$-metric spaces, rectangular metric spaces, and $\gamma$-generalized quasi metric spaces were respectively, introduced by Gahler [9], Dhage [7], Mustafa and Sims [10], Branciari [6], and Adewale et al. [1].

Motivated by these generalizations and the fact that in real life, two points in a space may be distinct, we present the notion of generalized rectangular metric space which extends a rectangular metric space. We also use an example to show that the newly introduced generalized rectangular metric space is better than rectangular metric space and different from $G$-metric spaces. Some fixed point theorems are stated and proved in this new space.

\section{Preliminary}

Mustafa and Sims define $G$-metric spaces as follows:

Definition 2.1 ([10]). For a non-empty set $X$ and a function $G: X^{3} \rightarrow[0, \infty)$ satisfying the following properties:

(i) $G(x, y, z)=0$ if and only if $x=y=z$;

(ii) $G(x, x, y)>0, \forall x, y \in X$, with $x \neq y$;

(iii) $G(x, x, y)<G(x, y, z), \forall x, y, z \in X$, with $z \neq y$; 
(iv) $G(x, y, z)=G(y, z, x)=G(x, z, y)=\ldots$;

(v) $\quad G(x, y, z) \leq G(x, a, a)+G(a, y, z), \quad \forall a, x, y, z \in X \quad$ (rectangle inequality).

The function $G$ is called a $G$-metric and $(X, G)$ is called a $G$-metric space.

Branciari also defined rectangular metric spaces as follows:

Definition 2.2 ([6]). For a non-empty set $X$ and a function $d: X^{2} \rightarrow[0, \infty)$ satisfying the following properties:

(i) $d(x, y)=0$ if and only if $x=y$ for all $x, y \in X$;

(ii) $d(x, y)=d(y, x), \quad \forall x y \in X$;

(iii) $\quad d(x, y) \leq d(x, u)+d(u, v)+d(v, y), \quad \forall x, y \in X$ and all distinct points $u, v \in X-\{x, y\}$.

The function $d$ is called a rectangular metric on $X$ and $(X, d)$ is called a rectangular metric space.

\section{Main Results}

We introduce the following:

Definition 3.1. For a non-empty set $X$ and a function $G: X^{3} \rightarrow$ $[0, \infty)$ satisfying the following properties:

(i) $G(x, y, z)=0$ if and only if $x=y=z$.

(ii) $G(x, x, y)>0, \forall x, y \in X$, with $x \neq y$.

(iii) $G(x, y, z)=G(x, z, y)=G(y, x, z)=\ldots$.

(iv) $G(x, y, z) \leq G(x, a, a)+G(a, b, b)+G(b, y, y)+G(y, y, z), \forall x, y, z \in X$ and all distinct points $a, b \in X-\{x, y, z\}$.

The function $G$ is called a generalized rectangular metric and $(X, G)$ is called a generalized rectangular metric space. 
Remark 3.2. If $y=z$ and we set $G(x, y, y)=d(x, y)$, Definition 3.1 reduces to rectangular metric space in [6].

Example 3.3. Let $X=R^{+} \cup\{0\}$ and define $G: X \times X \times X \rightarrow R^{+} \cup\{0\}$ $\bigcup\{0\}$ by

$$
G(x, y, z)=\left\{\begin{array}{lc}
0, & x=y=z \\
3, & \text { if } x \neq y \neq z \neq x \\
1, & \text { otherwise. }
\end{array}\right.
$$

Then $(X, G)$ is a generalized rectangular metric space but neither a $G$-metric space nor a rectangular metric space because

(i) $G(1,2,3)>G(1,2,2)+G(2,2,3)$,

(ii) the points $a$ and $b$ are distinct $(a, b \notin\{x, y, z\})$.

Definition 3.4. Let $(X, G)$ be a generalized rectangular metric space. For $x \in X, r>0$, the $G$-sphere with centre $x$ and radius $r$ is

$$
S_{G}(x, r)=\{z \in X: G(x, z, z)<r\} .
$$

Definition 3.5. Let $(X, G)$ be a generalized rectangular metric space. The sequence $\left\{x_{n}\right\} \subset X$ is $G$-convergent to $z$ if it converges to $z$ in the generalized rectangular metric spaces.

Definition 3.6. Let $(X, G)$ and $(\bar{X}, \bar{G})$ be two generalized rectangular metric spaces, a function $T: X \rightarrow \bar{X}$ is $G$-continuous at a point $x \in X$ if $T^{-1}\left(S_{\bar{G}}(T(x), r)\right) \in \tau(G)$, for all $r>0 . T$ is $G$-continuous if it is $G$-continuous at all points of $X$.

The following lemmas will be used in this work. 
Lemma 3.7. Let $(X, G)$ be a generalized rectangular metric space and $\left\{x_{n}\right\}$ be a sequence in $X$. Then $\left\{x_{n}\right\}$ converges to $x$ if and only if $G\left(x_{n}, x, x\right) \rightarrow 0$ as $n \rightarrow \infty$.

Lemma 3.8. Let $(X, G)$ be a generalized rectangular metric space and $\left\{x_{n}\right\}$ be a sequence in $X$. Then $\left\{x_{n}\right\}$ is said to be a Cauchy sequence if and only if $G\left(x_{n}, x_{m}, x_{l}\right) \rightarrow 0$ as $n, m, l \rightarrow \infty$.

Theorem 3.9. Let $X$ be a complete generalized rectangular metric space and $T: X \rightarrow X$ be a map for which there exist the real number, $k$ satisfying $0 \leq k<1$ such that for each pair $x, y, z \in X$.

$$
G(T x, T y, T z) \leq k G(x, y, z) .
$$

Then $T$ has a unique fixed point.

Proof. Considering (1),

$$
G(T x, T y, T y) \leq k G(x, y, y) .
$$

Suppose $T$ satisfies condition (2) and $x_{0} \in X$ be an arbitrary point and define the sequence $x_{n}$ by $x_{n}=T^{n} x_{0}$, then

$$
G\left(x_{n}, x_{n+1}, x_{n+1}\right)=G\left(T x_{n-1}, T x_{n}, T x_{n}\right) \leq k G\left(x_{n-1}, x_{n}, x_{n}\right) .
$$

Setting $g_{n}=G\left(x_{n}, x_{n+1}, x_{n+1}\right)$, we have

$$
g_{n} \leq k g_{n-1}
$$

We deduce that

$$
\begin{aligned}
& g_{n} \leq k g_{n-1}, \\
& g_{n} \leq k^{n} g_{0}, \quad \forall n \in N .
\end{aligned}
$$


Suppose there exists $n \in N$ such that $x_{0}=x_{n}$.

$$
\begin{aligned}
G\left(x_{0}, T x_{0}, T x_{0}\right) & =G\left(x_{n}, T x_{n}, T x_{n}\right), \\
G\left(x_{0}, x_{1}, x_{1}\right) & =G\left(x_{n}, x_{n+1}, x_{n+1}\right), \\
g_{0} & =g_{n} \\
g_{0} & \leq k^{n} g_{0}
\end{aligned}
$$

Contradiction since $k<1$. Hence $\forall n \in N, x_{0} \neq x_{n}$. Repeating this argument, we have that $\forall n, m \in N$ with $n \neq m, x_{n} \neq x_{m}$. Then the terms of the sequence $\left\{x_{n}\right\}$ are distinct.

By repeated use of (iv) in Definition 3.1 and all distinct points $x_{n+1}, x_{n+2}, \ldots, x_{m-1}$ with $m>n$, we have the following for all odd $m-n$ :

$$
\begin{aligned}
G\left(x_{n}, x_{m}, x_{m}\right) \leq & G\left(x_{n}, x_{n+1}, x_{n+1}\right)+G\left(x_{n+1}, x_{n+2}, x_{n+2}\right) \\
& +G\left(x_{n+2}, x_{m}, x_{m}\right) \\
\leq & g_{n}+g_{n+1}+G\left(x_{n+2}, x_{m}, x_{m}\right) \\
\leq & g_{n}+g_{n+1}+g_{n+2}+g_{n+3}+G\left(x_{n+4}, x_{m}, x_{m}\right) \\
\leq & \sum_{i=n}^{n+3} g_{i}+G\left(x_{n+4}, x_{m}, x_{m}\right) \\
\leq & \sum_{i=n}^{m-1} g_{i} \leq \sum_{i=n}^{\infty} g_{i} .
\end{aligned}
$$

Similarly, if $m-n \geq 4$ is even, we have

$$
G\left(x_{n}, x_{m}, x_{m}\right) \leq \sum_{i=n}^{m-3} g_{i}+G\left(x_{m-2}, x_{m}, x_{m}\right) .
$$


From (4) and (5), we have

$$
\begin{aligned}
G\left(x_{n}, x_{m}, x_{m}\right) & \leq k^{n} g_{0}+k^{n+1} g_{0}+k^{n+2} g_{0}+\ldots+k^{m-2} g_{0}+k^{m-1} g_{0} \\
& \leq\left[k^{n}+k^{n+1}+k^{n+2}+k^{n+3}+\ldots+k^{m-1}\right] g_{0} \\
& \leq k^{n}\left[1+k+k^{2}+k^{3}+k^{4}+\ldots+k^{m-n-1}\right] g_{0} \\
& \leq k^{n}(1-k)^{-1} g_{0} .
\end{aligned}
$$

From (4) and (6), we have

$$
\begin{aligned}
G\left(x_{n}, x_{m}, x_{m}\right) & \leq k^{n}(1-k)^{-1} g_{0}+G\left(x_{m-2}, x_{m}, x_{m}\right) \\
& \leq k^{n}(1-k)^{-1} g_{0}+k^{m-2} G\left(x_{0}, x_{2}, x_{2}\right) .
\end{aligned}
$$

Taking the limit of $G\left(x_{n}, x_{m}, x_{m}\right)$ as $n, m \rightarrow \infty$, we have

$$
\lim _{n, m \rightarrow \infty} G\left(x_{n}, x_{m}, x_{m}\right)=0 .
$$

For $n, m, l \in N$ with $n>m>l$,

$$
\begin{aligned}
G\left(x_{n}, x_{m}, x_{l}\right) \leq & G\left(x_{n}, x_{n-1}, x_{n-1}\right)+G\left(x_{n-1}, x_{n-2}, x_{n-2}\right) \\
& +G\left(x_{n-2}, x_{m}, x_{m}\right)+G\left(x_{m}, x_{m}, x_{l}\right) .
\end{aligned}
$$

Taking the limit of $G\left(x_{n}, x_{m}, x_{l}\right)$ as $n, m, l \rightarrow \infty$, we have

$$
\lim _{n, m, l \rightarrow \infty} G\left(x_{n}, x_{m}, x_{l}\right)=0 .
$$

So, $\left\{x_{n}\right\}$ is a $G$-Cauchy sequence.

By completeness of $(X, G)$, there exist $u \in X$ such that $x_{n}$ is $G$-convergent to $u$.

Suppose $T u \neq u$

$$
G\left(x_{n}, T u, T u\right) \leq k G\left(x_{n-1}, u, u\right) .
$$


Taking the limit $n \rightarrow \infty$ and using the fact that $T$ is $G$-continuous in its variables, we get

$$
G(u, T u, T u) \leq k G(u, u, u) .
$$

Hence,

$$
G(u, T u, T u) \leq 0
$$

This is a contradiction. So, $T u=u$. To show the uniqueness, suppose $v \neq u$ is such that $T v=v$, then

$$
G(T u, T v, T v) \leq k G(u, v, v) .
$$

Since $T u=u$ and $T v=v$, we have

$$
G(u, v, v) \leq 0,
$$

which implies that $v=u$.

Remark 3.10. Let $(X, G)$ be a rectangular $G$-metric space and $d: X \times X \rightarrow[0, \infty)$ be a function defined by $G(x, y, y)=d(x, y)$, then Theorem 2.9 reduces to Banach contraction principle in rectangularmetric space (an analogue of Banach contraction principle in metric space).

Theorem 3.11. Let $X$ be a complete generalized rectangular metric space and $T: X \rightarrow X$ be a map for which there exist the real number $b$ satisfying $0 \leq b<\frac{1}{3}$ such that for each pair $x, y, z \in X$.

$$
G(T x, T y, T z) \leq b[G(x, T x, T x)+G(y, T y, T y)+G(z, T z, T z)] .
$$

Then $T$ has a unique fixed point.

Proof. Considering (17),

$$
G(T x, T y, T y) \leq b[G(x, T x, T x)+G(y, T y, T y)+G(z, T z, T z)] .
$$


Suppose $T$ satisfies condition (18) and $x_{0} \in X$ be an arbitrary point and define the sequence $x_{n}$ by $x_{n}=T^{n} x_{0}$, then we have

$$
\begin{aligned}
G\left(x_{n}, x_{n+1}, x_{n+1}\right) \leq & b\left[G\left(x_{n-1}, x_{n}, x_{n}\right)+G\left(x_{n}, x_{n+1}, x_{n+1}\right)\right. \\
& \left.+G\left(x_{n}, x_{n+1}, x_{n+1}\right)\right] .
\end{aligned}
$$

We deduce that

$$
G\left(x_{n}, x_{n+1}, x_{n+1}\right) \leq \frac{b}{1-2 b} G\left(x_{n-1}, x_{n}, x_{n}\right) .
$$

Let $p=\frac{b}{1-2 b}<1$

$$
\begin{aligned}
G\left(x_{n}, x_{n+1}, x_{n+1}\right) & \leq p G\left(x_{n-1}, x_{n}, x_{n}\right) \\
& \leq p^{2} G\left(x_{n-2}, x_{n-1}, x_{n-1}\right) \\
G\left(x_{n}, x_{n+1}, x_{n+1}\right) & \leq p^{3} G\left(x_{n-3}, x_{n-2}, x_{n-2}\right) \\
G\left(x_{n}, x_{n+1}, x_{n+1}\right) & \leq p^{n} G\left(x_{0}, x_{1}, x_{1}\right) \\
g_{n} & \leq p^{n} g_{0} .
\end{aligned}
$$

Suppose there exists $n \in N$ such that $x_{0}=x_{n}$.

$$
\begin{aligned}
G\left(x_{0}, T x_{0}, T x_{0}\right) & =G\left(x_{n}, T x_{n}, T x_{n}\right), \\
G\left(x_{0}, x_{1}, x_{1}\right) & =G\left(x_{n}, x_{n+1}, x_{n+1}\right), \\
g_{0} & =g_{n} \\
g_{0} & \leq k^{n} g_{0} .
\end{aligned}
$$

Contradiction since $k<1$. Hence $\forall n \in N, x_{0} \neq x_{n}$. Repeating this argument, we have that $\forall n, m \in N$ with $n \neq m, x_{n} \neq x_{m}$. Then the terms of the sequence $\left\{x_{n}\right\}$ are distinct. 
By repeated use of (iv) in Definition 3.1 and all distinct points $x_{n+1}, x_{n+2}, \ldots, x_{m-1}$, we have the following for all odd $m-n$ :

$$
\begin{aligned}
G\left(x_{n}, x_{m}, x_{m}\right) \leq & G\left(x_{n}, x_{n+1}, x_{n+1}\right)+G\left(x_{n+1}, x_{n+2}, x_{n+2}\right) \\
& +G\left(x_{n+2}, x_{m}, x_{m}\right) \\
\leq & g_{n}+g_{n+1}+G\left(x_{n+2}, x_{m}, x_{m}\right) \\
\leq & g_{n}+g_{n+1}+g_{n+2}+g_{n+3}+G\left(x_{n+4}, x_{m}, x_{m}\right) \\
\leq & \sum_{i=n}^{n+3} g_{i}+G\left(x_{n+4}, x_{m}, x_{m}\right) \\
\leq & \sum_{i=n}^{m-1} g_{i} \leq \sum_{i=n}^{\infty} g_{i} .
\end{aligned}
$$

Similarly, if $m-n \geq 4$ is even, we have

$$
G\left(x_{n}, x_{m}, x_{m}\right) \leq \sum_{i=n}^{m-3} g_{i}+G\left(x_{m-2}, x_{m}, x_{m}\right) .
$$

From (19) and (20), we have

$$
\begin{aligned}
G\left(x_{n}, x_{m}, x_{m}\right) & \leq p^{n} g_{0}+p^{n+1} g_{0}+p^{n+2} g_{0}+\ldots+p^{m-2} g_{0}+p^{m-1} g_{0} \\
& \leq\left[p^{n}+p^{n+1}+p^{n+2}+p^{n+3}+\ldots+p^{m-1}\right] g_{0} \\
& \leq p^{n}\left[1+p+p^{2}+p^{3}+p^{4}+\ldots+p^{m-n-1}\right] g_{0} \\
& \leq p^{n}(1-p)^{-1} g_{0} .
\end{aligned}
$$

From (19) and (21), we have

$$
\begin{aligned}
G\left(x_{n}, x_{m}, x_{m}\right) & \leq p^{n}(1-p)^{-1} g_{0}+G\left(x_{m-2}, x_{m}, x_{m}\right) \\
& \leq p^{n}(1-p)^{-1} g_{0}+p^{m-2} G\left(x_{0}, x_{2}, x_{2}\right) .
\end{aligned}
$$


Taking the limit of $G\left(x_{n}, x_{m}, x_{m}\right)$ as $n, m \rightarrow \infty$, we have

$$
\lim _{n, m \rightarrow \infty} G\left(x_{n}, x_{m}, x_{m}\right)=0 .
$$

For $n, m, l \in N$ with $n>m>l$,

$$
\begin{aligned}
G\left(x_{n}, x_{m}, x_{l}\right) \leq & G\left(x_{n}, x_{n-1}, x_{n-1}\right)+G\left(x_{n-1}, x_{n-2}, x_{n-2}\right) \\
& +G\left(x_{n-2}, x_{m}, x_{m}\right)+G\left(x_{m}, x_{m}, x_{l}\right) .
\end{aligned}
$$

Taking the limit of $G\left(x_{n}, x_{m}, x_{l}\right)$ as $n, m, l \rightarrow \infty$, we have

$$
\lim _{n, m, l \rightarrow \infty} G\left(x_{n}, x_{m}, x_{l}\right)=0 .
$$

So, $x_{n}$ is a $G$-Cauchy sequence.

By completeness of $(X, G)$, there exist $u \in X$ such that $x_{n}$ is $G$-convergent to $u$.

$$
\begin{aligned}
\text { Suppose } T u \neq u & \\
G\left(x_{n}, T u, T u\right) & \leq b\left[G\left(x_{n-1}, x_{n}, x_{n}\right)+G(u, T u, T u)+G(u, T u, T u)\right] \\
& \leq b\left[G\left(x_{n-1}, x_{n}, x_{n}\right)+2 G(u, T u, T u)\right]
\end{aligned}
$$

Taking the limit as $n \rightarrow \infty$ and using the fact that $T$ is $G$-continuous in its variables, we get

$$
G(u, T u, T u) \leq 2 b G(u, T u, T u) .
$$

Hence,

$$
G(u, T u, T u) \leq 0 .
$$

This is a contradiction. So, $T u=u$. To show the uniqueness, suppose $v \neq u$ is such that $T v=v$, then

$$
G(T u, T v, T v) \leq b[G(u, T u, T u)+G(v, T v, T v)+G(v, T v, T v)] .
$$

Since $T u=u$ and $T v=v$, we have

$$
G(u, v, v) \leq 0,
$$

which implies that $v=u$. 
Remark 3.12. Let $(X, G)$ be a rectangular $G$-metric space and $d: X \times X \rightarrow[0, \infty)$ be a function defined by $G(x, y, y)=d(x, y)$, then Theorem 3.11 reduces to Kannan's fixed point theorem in rectangularmetric space (an analogue of Kannan's fixed point theorem in metric space).

Theorem 3.13. Let $X$ be a complete generalized rectangular metric space and $T: X \rightarrow X$ be a map for which there exists real numbers $a, b, c$ satisfying $0 \leq a<1,0 \leq b<\frac{1}{2}, 0 \leq c<\frac{1}{2}$ with $\delta=\max \left\{a, \frac{b}{1-b}, \frac{c}{1-c}\right\}$ and

$$
\phi(t)= \begin{cases}0, & \text { if } \quad t=0, \\ \frac{t}{3}, & \text { if } \quad t \neq 0,\end{cases}
$$

such that for each pair $x, y, z \in X$.

$$
G(T x, T y, T z) \leq \phi(\delta G(x, y, z)+2 \delta G(x, T x, T x)) .
$$

Then $T$ has a unique fixed point.

Proof. Considering (32),

$$
G(T x, T y, T y) \leq \phi(\delta G(x, y, y)+2 \delta G(x, T x, T x))
$$

Suppose $T$ satisfies condition (33) and $x_{0} \in X$ be an arbitrary point and define the sequence $x_{n}$ by $x_{n}=T^{n} x_{0}$, then

$$
\begin{aligned}
G\left(x_{n}, x_{n+1}, x_{n+1}\right) & =G\left(T x_{n-1}, T x_{n}, T x_{n}\right) \\
& \leq \phi\left(\delta G\left(x_{n-1}, x_{n}, x_{n}\right)+2 \delta G\left(x_{n-1}, x_{n}, x_{n}\right)\right) \\
& \leq \phi\left(3 \delta G\left(x_{n-1}, x_{n}, x_{n}\right)\right) \\
& \leq \delta G\left(x_{n-1}, x_{n}, x_{n}\right) .
\end{aligned}
$$


Setting $g_{n}=G\left(x_{n}, x_{n+1}, x_{n+1}\right)$, then

$$
g_{n} \leq \delta g_{n-1} .
$$

Deducing

$$
\begin{aligned}
& g_{n} \leq \delta g_{n-1}, \\
& g_{n} \leq \delta^{n} g_{0}, \quad \forall n \in \mathbb{N} \cup\{0\} .
\end{aligned}
$$

Suppose there exists $n \in N$ such that $x_{0}=x_{n}$.

$$
\begin{aligned}
G\left(x_{0}, T x_{0}, T x_{0}\right) & =G\left(x_{n}, T x_{n}, T x_{n}\right), \\
G\left(x_{0}, x_{1}, x_{1}\right) & =G\left(x_{n}, x_{n+1}, x_{n+1}\right), \\
g_{0} & =g_{n} \\
g_{0} & \leq \delta^{n} g_{0} .
\end{aligned}
$$

Contradiction since $\delta<1$. Hence $\forall n \in \mathbb{N} \cup\{0\}, x_{0} \neq x_{n}$. Repeating this argument, $\forall n, m \in \mathbb{N} \cup\{0\}$ with $n \neq m, x_{n} \neq x_{m}$. Then the terms of the sequence $\left\{x_{n}\right\}$ are distinct.

By repeated use of (iv) in Definition 3.1 and all distinct points $x_{n+1}, x_{n+2}, \ldots, x_{m-1}$ with $m>n$, we have the following for all odd $m-n$ :

$$
\begin{aligned}
G\left(x_{n}, x_{m}, x_{m}\right) \leq & G\left(x_{n}, x_{n+1}, x_{n+1}\right)+G\left(x_{n+1}, x_{n+2}, x_{n+2}\right) \\
& +G\left(x_{n+2}, x_{m}, x_{m}\right) \\
\leq & g_{n}+g_{n+1}+G\left(x_{n+2}, x_{m}, x_{m}\right) \\
\leq & g_{n}+g_{n+1}+g_{n+2}+g_{n+3}+G\left(x_{n+4}, x_{m}, x_{m}\right) \\
\leq & \sum_{i=n}^{n+3} g_{i}+G\left(x_{n+4}, x_{m}, x_{m}\right) \\
\leq & \sum_{i=n}^{m-1} g_{i} \leq \sum_{i=n}^{\infty} g_{i} .
\end{aligned}
$$


Similarly, if $m-n \geq 4$ is even, we have

$$
G\left(x_{n}, x_{m}, x_{m}\right) \leq \sum_{i=n}^{m-3} g_{i}+G\left(x_{m-2}, x_{m}, x_{m}\right) .
$$

From (34) and (36), the following are obtained.

$$
\begin{aligned}
G\left(x_{n}, x_{m}, x_{m}\right) & \leq \delta^{n} g_{0}+\delta^{n+1} g_{0}+\delta^{n+2} g_{0}+\ldots+\delta^{m-2} g_{0}+\delta^{m-1} g_{0} \\
& \leq\left[\delta^{n}+\delta^{n+1}+\delta^{n+2}+\delta^{n+3}+\ldots+\delta^{m-1}\right] g_{0} \\
& \leq \delta^{n}\left[1+\delta+\delta^{2}+\delta^{3}+\delta^{4}+\ldots+\delta^{m-n-1}\right] g_{0} \\
& \leq \delta^{n}(1-\delta)^{-1} g_{0} .
\end{aligned}
$$

From (34) and (37), we have

$$
\begin{aligned}
G\left(x_{n}, x_{m}, x_{m}\right) & \leq \delta^{n}(1-\delta)^{-1} g_{0}+G\left(x_{m-2}, x_{m}, x_{m}\right) \\
& \leq \delta^{n}(1-\delta)^{-1} g_{0}+\delta^{m-2} G\left(x_{0}, x_{2}, x_{2}\right) .
\end{aligned}
$$

Taking the limit of $G\left(x_{n}, x_{m}, x_{m}\right)$ as $n, m \rightarrow \infty$,

$$
\lim _{n, m \rightarrow \infty} G\left(x_{n}, x_{m}, x_{m}\right)=0 .
$$

For $n, m, l \in \mathbb{N} \cup\{0\}$ with $n>m>l$,

$$
\begin{aligned}
G\left(x_{n}, x_{m}, x_{l}\right) \leq & G\left(x_{n}, x_{n-1}, x_{n-1}\right)+G\left(x_{n-1}, x_{n-2}, x_{n-2}\right) \\
& +G\left(x_{n-2}, x_{m}, x_{m}\right)+G\left(x_{m}, x_{m}, x_{l}\right) .
\end{aligned}
$$

Taking the limit of $G\left(x_{n}, x_{m}, x_{l}\right)$ as $n, m, l \rightarrow \infty$,

$$
\lim _{n, m, l \rightarrow \infty} G\left(x_{n}, x_{m}, x_{l}\right)=0 .
$$

So, $\left\{x_{n}\right\}$ is a $G$-Cauchy sequence. 
By completeness of $(X, G)$, there exist $u \in X$ such that $x_{n}$ is $G$-convergent to $u$.

$$
\begin{aligned}
& \text { Suppose } T u \neq u \\
& \qquad G\left(x_{n}, T u, T u\right) \leq \phi\left(\delta G\left(x_{n-1}, u, u\right)+2 \delta G\left(x_{n-1}, x_{n}, x_{n}\right)\right) .
\end{aligned}
$$

Taking the limit as $n \rightarrow \infty$ and using the fact that $T$ is $G$-continuous in its variables,

$$
G(u, T u, T u) \leq \phi(\delta G(u, u, u)+2 \delta G(u, u, u)) .
$$

Hence,

$$
G(u, T u, T u) \leq 0 .
$$

This is a contradiction. So, $T u=u$. To show the uniqueness, suppose $v \neq u$ is such that $T v=v$, then

$$
G(T u, T v, T v) \leq \phi(\delta G(u, v, v)+2 \delta G(u, T u, T u)) .
$$

Since $T u=u$ and $T v=v$, then

$$
G(u, v, v) \leq 0
$$

which implies that $v=u$.

Remark 3.14. Let $(X, G)$ be a rectangular $G$-metric space and $d: X \times X \rightarrow[0, \infty)$ be a function defined by $G(x, y, y)=d(x, y)$ with $\phi(t)=t$, then Theorem 3.13 reduces to Zamfirescu's fixed point theorem in rectangular-metric space (an analogue of Zamfirescu's fixed point theorem in metric space).

Theorem 3.15. Let $X$ be a complete generalized rectangular metric space and $T: X \rightarrow X$ be a map for which there exists real numbers $a, b, c$ satisfying $0 \leq a<1,0 \leq b<\frac{1}{2}, 0 \leq c<\frac{1}{2} \quad$ with $\delta=\max \left\{a, \frac{b}{1-b}, \frac{c}{1-c}\right\}$ such that for each pair $x, y, z \in X$.

$$
G(T x, T y, T z) \leq \delta G(x, y, z)+\vartheta(2 \delta G(T x, y, z)),
$$


where $\delta \in[0,1)$ and function $\vartheta: \mathbb{R}^{+} \rightarrow \mathbb{R}^{+}$with $\vartheta(t)=t$ a monotone increasing sequence. Then $T$ has a unique fixed point.

Proof. Considering (48),

$$
G(T x, T y, T y) \leq \delta G(x, y, y)+\chi(2 \delta G(T x, y, y)) .
$$

Suppose $T$ satisfies condition (49) and $x_{0} \in X$ be an arbitrary point and define the sequence $x_{n}$ by $x_{n}=T^{n} x_{0}$, then

$$
\begin{aligned}
G\left(x_{n}, x_{n+1}, x_{n+1}\right) & =G\left(T x_{n-1}, T x_{n}, T x_{n}\right) \\
& \leq \delta G\left(x_{n-1}, x_{n}, x_{n}\right)+\gamma\left(2 \delta G\left(x_{n}, x_{n}, x_{n}\right)\right) \\
& \leq \delta G\left(x_{n-1}, x_{n}, x_{n}\right) .
\end{aligned}
$$

Setting $g_{n}=G\left(x_{n}, x_{n+1}, x_{n+1}\right)$, then

$$
g_{n} \leq \delta g_{n-1}
$$

Deducing

$$
\begin{aligned}
& g_{n} \leq \delta g_{n-1}, \\
& g_{n} \leq \delta^{n} g_{0}, \quad \forall n \in \mathbb{N} \cup\{0\} .
\end{aligned}
$$

Suppose there exists $n \in N$ such that $x_{0}=x_{n}$.

$$
\begin{aligned}
G\left(x_{0}, T x_{0}, T x_{0}\right) & =G\left(x_{n}, T x_{n}, T x_{n}\right), \\
G\left(x_{0}, x_{1}, x_{1}\right) & =G\left(x_{n}, x_{n+1}, x_{n+1}\right), \\
g_{0} & =g_{n} \\
g_{0} & \leq \delta^{n} g_{0} .
\end{aligned}
$$

Contradiction since $\delta<1$. Hence $\forall n \in \mathbb{N} \cup\{0\}, x_{0} \neq x_{n}$. Repeating this argument, $\forall n, m \in \mathbb{N} \cup\{0\}$ with $n \neq m, x_{n} \neq x_{m}$. Then the terms of the sequence $\left\{x_{n}\right\}$ are distinct. 
By repeated use of (iv) in Definition 3.1 and all distinct points $x_{n+1}, x_{n+2}, \ldots, x_{m-1}$ with $m>n$, we have the following for all odd $m-n$ :

$$
\begin{aligned}
G\left(x_{n}, x_{m}, x_{m}\right) \leq & G\left(x_{n}, x_{n+1}, x_{n+1}\right)+G\left(x_{n+1}, x_{n+2}, x_{n+2}\right) \\
& +G\left(x_{n+2}, x_{m}, x_{m}\right) \\
\leq & g_{n}+g_{n+1}+G\left(x_{n+2}, x_{m}, x_{m}\right) \\
\leq & g_{n}+g_{n+1}+g_{n+2}+g_{n+3}+G\left(x_{n+4}, x_{m}, x_{m}\right) \\
\leq & \sum_{i=n}^{n+3} g_{i}+G\left(x_{n+4}, x_{m}, x_{m}\right) \\
\leq & \sum_{i=n}^{m-1} g_{i} \leq \sum_{i=n}^{\infty} g_{i} .
\end{aligned}
$$

Similarly, if $m-n \geq 4$ is even, we have

$$
G\left(x_{n}, x_{m}, x_{m}\right) \leq \sum_{i=n}^{m-3} g_{i}+G\left(x_{m-2}, x_{m}, x_{m}\right) .
$$

From (50) and (52), the following are obtained:

$$
\begin{aligned}
G\left(x_{n}, x_{m}, x_{m}\right) & \leq \delta^{n} g_{0}+\delta^{n+1} g_{0}+\delta^{n+2} g_{0}+\ldots+\delta^{m-2} g_{0}+\delta^{m-1} g_{0} \\
& \leq\left[\delta^{n}+\delta^{n+1}+\delta^{n+2}+\delta^{n+3}+\ldots+\delta^{m-1}\right] g_{0} \\
& \leq \delta^{n}\left[1+\delta+\delta^{2}+\delta^{3}+\delta^{4}+\ldots+\delta^{m-n-1}\right] g_{0} \\
& \leq \delta^{n}(1-\delta)^{-1} g_{0} .
\end{aligned}
$$

From (50) and (53), we have

$$
\begin{aligned}
G\left(x_{n}, x_{m}, x_{m}\right) & \leq \delta^{n}(1-\delta)^{-1} g_{0}+G\left(x_{m-2}, x_{m}, x_{m}\right) \\
& \leq \delta^{n}(1-\delta)^{-1} g_{0}+\delta^{m-2} G\left(x_{0}, x_{2}, x_{2}\right) .
\end{aligned}
$$


Taking the limit of $G\left(x_{n}, x_{m}, x_{m}\right)$ as $n, m \rightarrow \infty$,

$$
\lim _{n, m \rightarrow \infty} G\left(x_{n}, x_{m}, x_{m}\right)=0 .
$$

For $n, m, l \in \mathbb{N} \cup\{0\}$ with $n>m>l$,

$$
\begin{aligned}
G\left(x_{n}, x_{m}, x_{l}\right) \leq & G\left(x_{n}, x_{n-1}, x_{n-1}\right)+G\left(x_{n-1}, x_{n-2}, x_{n-2}\right) \\
& +G\left(x_{n-2}, x_{m}, x_{m}\right)+G\left(x_{m}, x_{m}, x_{l}\right) .
\end{aligned}
$$

Taking the limit of $G\left(x_{n}, x_{m}, x_{l}\right)$ as $n, m, l \rightarrow \infty$,

$$
\lim _{n, m, l \rightarrow \infty} G\left(x_{n}, x_{m}, x_{l}\right)=0 .
$$

So, $\left\{x_{n}\right\}$ is a $G$-Cauchy sequence.

By completeness of $(X, G)$, there exist $u \in X$ such that $x_{n}$ is $G$-convergent to $u$.

Suppose $T u \neq u$

$$
G\left(x_{n}, T u, T u\right) \leq \delta G\left(x_{n-1}, u, u\right)+\downarrow\left(2 \delta G\left(x_{n}, u, u\right)\right) .
$$

Taking the limit as $n \rightarrow \infty$ and using the fact that $T$ is $G$-continuous in its variables,

$$
G(u, T u, T u) \leq \delta G(u, u, u)+\vartheta(2 \delta G(u, u, u)) .
$$

Hence,

$$
G(u, T u, T u) \leq 0 .
$$

This is a contradiction. So, $T u=u$. To show the uniqueness, suppose $v \neq u$ is such that $T v=v$, then

$$
G(T u, T v, T v) \leq \delta G(u, v, v)+\vartheta(2 \delta G(T u, v, v)) .
$$

Since $T u=u$ and $T v=v$, then

$$
G(u, v, v) \leq 0,
$$

which implies that $v=u$. 
Remark 3.16. Let $(X, G)$ be a rectangular $G$-metric space and $d: X \times X \rightarrow[0, \infty)$ be a function defined by $G(x, y, y)=d(x, y)$ with $\vartheta(2 \delta G(T x, y, z))=0$, then Theorem 3.15 reduces to Banach contraction principle in rectangular-metric space (an analogue of Banach contraction principle in metric space).

Example 3.17. Let $X=\mathbb{R}$ endowed with a generalized rectangular metric $G(x, y, z)=\max \{|x-y|,|x-z|,|y-z|\}$ for all $x, y, z \in X$. Define the mapping $T: X \rightarrow X$ by

$$
T(x)= \begin{cases}2, & \text { if } \quad x \geq 1, \\ 1, & \text { if } \quad x \in[0,1), \\ 0, & \text { otherwise. }\end{cases}
$$

It is observed that Banach contraction principle in $G$-metric space introduced by Mustafa and Sims [10] cannot be applied in this case because

$$
\begin{aligned}
G(T(-1), T(1), T(3)) & =G(0,2,2) \\
& =\max \{|0-2|,|0-2|,|2-2|\} \\
& =\max \{2,2,0\} \\
& =2 \\
& >k G(-1,1,3) \\
& >k \max \{|-1-1|,|-1-3|,|1-3|\} \\
& >k \max \{2,4,2\} \\
& >4 k=4 \times 0.25=1,0.25 \in[0,1) .
\end{aligned}
$$


With the newly introduced contractive mapping, the above case can be taken care off. As a matter of fact, for all $x, y, z \in X$,

$$
G(T x, T y, T z) \leq \delta G(x, y, z)+\chi(2 \delta G(T x, y, z)) .
$$

Since $\delta=\max \left\{a, \frac{b}{1-b}, \frac{c}{1-c}\right\}, 0 \leq G(T x, T y, T z) \leq 2$ and $\emptyset(t)=t$.

Theorem 3.18. Let $X$ be a complete generalized rectangular metric space and $T: X \rightarrow X$ be a map for which there exists real numbers $a, b, c$ satisfying $0 \leq a<1,0 \leq b<\frac{1}{2}, 0 \leq c<\frac{1}{2}$ with $\delta=\max \left\{a, \frac{b}{1-b}, \frac{c}{1-c}\right\}$ such that for each pair $x, y, z \in X$.

$$
G(T x, T y, T z) \leq \phi(\delta G(x, y, z))+\downarrow(2 \delta G(x, T x, T x)),
$$

where $\delta \in[0,1)$ and functions $\phi, \wp: \mathbb{R}^{+} \rightarrow \mathbb{R}^{+}$with $\quad x(t)=\frac{t}{4}$ and $\phi(t)=\frac{t}{2}$ a monotone increasing sequences. Then $T$ has a unique fixed point.

Proof. Considering (64),

$$
G(T x, T y, T y) \leq \phi(\delta G(x, y, y))+\gamma(2 \delta G(x, T x, T x)) .
$$

Suppose $T$ satisfies condition (65) and $x_{0} \in X$ be an arbitrary point and define the sequence $x_{n}$ by $x_{n}=T^{n} x_{0}$, then

$$
\begin{aligned}
G\left(x_{n}, x_{n+1}, x_{n+1}\right) & =G\left(T x_{n-1}, T x_{n}, T x_{n}\right) \\
& \leq \phi\left(\delta G\left(x_{n-1}, x_{n}, x_{n}\right)\right)+\emptyset\left(2 \delta G\left(x_{n-1}, x_{n}, x_{n}\right)\right) \\
& \leq \delta G\left(x_{n-1}, x_{n}, x_{n}\right) .
\end{aligned}
$$

Setting $g_{n}=G\left(x_{n}, x_{n+1}, x_{n+1}\right)$, then

$$
g_{n} \leq \delta g_{n-1} .
$$


Deducing

$$
\begin{aligned}
& g_{n} \leq \delta g_{n-1}, \\
& g_{n} \leq \delta^{n} g_{0}, \quad \forall n \in \mathbb{N} \cup\{0\} .
\end{aligned}
$$

Suppose there exists $n \in N$ such that $x_{0}=x_{n}$.

$$
\begin{aligned}
G\left(x_{0}, T x_{0}, T x_{0}\right) & =G\left(x_{n}, T x_{n}, T x_{n}\right), \\
G\left(x_{0}, x_{1}, x_{1}\right) & =G\left(x_{n}, x_{n+1}, x_{n+1}\right), \\
g_{0} & =g_{n} \\
g_{0} & \leq \delta^{n} g_{0} .
\end{aligned}
$$

Contradiction since $\delta<1)$. Hence $\forall n \in \mathbb{N} \cup\{0\}, x_{0} \neq x_{n}$. Repeating this argument, $\forall n, m \in \mathbb{N} \cup\{0\}$ with $n \neq m, x_{n} \neq x_{m}$. Then the terms of the sequence $\left\{x_{n}\right\}$ are distinct.

By repeated use of (iv) in Definition 3.1 and all distinct points $x_{n+1}, x_{n+2}, \ldots, x_{m-1}$ with $m>n$, we have the following for all odd $m-n$ :

$$
\begin{aligned}
G\left(x_{n}, x_{m}, x_{m}\right) \leq & G\left(x_{n}, x_{n+1}, x_{n+1}\right)+G\left(x_{n+1}, x_{n+2}, x_{n+2}\right) \\
& +G\left(x_{n+2}, x_{m}, x_{m}\right) \\
\leq & g_{n}+g_{n+1}+G\left(x_{n+2}, x_{m}, x_{m}\right) \\
\leq & g_{n}+g_{n+1}+g_{n+2}+g_{n+3}+G\left(x_{n+4}, x_{m}, x_{m}\right) \\
\leq & \sum_{i=n}^{n+3} g_{i}+G\left(x_{n+4}, x_{m}, x_{m}\right) \\
\leq & \sum_{i=n}^{m-1} g_{i} \leq \sum_{i=n}^{\infty} g_{i} .
\end{aligned}
$$


Similarly, if $m-n \geq 4$ is even, we have

$$
G\left(x_{n}, x_{m}, x_{m}\right) \leq \sum_{i=n}^{m-3} g_{i}+G\left(x_{m-2}, x_{m}, x_{m}\right) .
$$

From (66) and (68), the following are obtained:

$$
\begin{aligned}
G\left(x_{n}, x_{m}, x_{m}\right) & \leq \delta^{n} g_{0}+\delta^{n+1} g_{0}+\delta^{n+2} g_{0}+\ldots+\delta^{m-2} g_{0}+\delta^{m-1} g_{0} \\
& \leq\left[\delta^{n}+\delta^{n+1}+\delta^{n+2}+\delta^{n+3}+\ldots+\delta^{m-1}\right] g_{0} \\
& \leq \delta^{n}\left[1+\delta+\delta^{2}+\delta^{3}+\delta^{4}+\ldots+\delta^{m-n-1}\right] g_{0} \\
& \leq \delta^{n}(1-\delta)^{-1} g_{0} .
\end{aligned}
$$

From (66) and (69), we have

$$
\begin{aligned}
G\left(x_{n}, x_{m}, x_{m}\right) & \leq \delta^{n}(1-\delta)^{-1} g_{0}+G\left(x_{m-2}, x_{m}, x_{m}\right) \\
& \leq \delta^{n}(1-\delta)^{-1} g_{0}+\delta^{m-2} G\left(x_{0}, x_{2}, x_{2}\right) .
\end{aligned}
$$

Taking the limit of $G\left(x_{n}, x_{m}, x_{m}\right)$ as $n, m \rightarrow \infty$,

$$
\lim _{n, m \rightarrow \infty} G\left(x_{n}, x_{m}, x_{m}\right)=0 .
$$

For $n, m, l \in \mathbb{N} \cup\{0\}$ with $n>m>l$,

$$
\begin{aligned}
G\left(x_{n}, x_{m}, x_{l}\right) \leq & G\left(x_{n}, x_{n-1}, x_{n-1}\right)+G\left(x_{n-1}, x_{n-2}, x_{n-2}\right) \\
& +G\left(x_{n-2}, x_{m}, x_{m}\right)+G\left(x_{m}, x_{m}, x_{l}\right) .
\end{aligned}
$$

Taking the limit of $G\left(x_{n}, x_{m}, x_{l}\right)$ as $n, m, l \rightarrow \infty$,

$$
\lim _{n, m, l \rightarrow \infty} G\left(x_{n}, x_{m}, x_{l}\right)=0 .
$$

So, $\left\{x_{n}\right\}$ is a $G$-Cauchy sequence. 
By completeness of $(X, G)$, there exist $u \in X$ such that $x_{n}$ is $G$-convergent to $u$.

$$
\begin{aligned}
& \text { Suppose } T u \neq u \\
& \qquad G\left(x_{n}, T u, T u\right) \leq \phi\left(\delta G\left(x_{n-1}, u, u\right)\right)+\vartheta\left(2 \delta G\left(x_{n-1}, x_{n}, x_{n}\right)\right) .
\end{aligned}
$$

Taking the limit as $n \rightarrow \infty$ and using the fact that $T$ is $G$-continuous in its variables,

$$
G(u, T u, T u) \leq \phi(\delta G(u, u, u))+\vartheta(2 \delta G(u, u, u)) .
$$

Hence,

$$
G(u, T u, T u) \leq 0
$$

This is a contradiction. So, $T u=u$. To show the uniqueness, suppose $v \neq u$ is such that $T v=v$, then

$$
G(T u, T v, T v) \leq \phi(\delta G(u, v, v))+\vartheta(2 \delta G(u, T u, T u)) .
$$

Since $T u=u$ and $T v=v$, then

$$
G(u, v, v) \leq 0,
$$

which implies that $v=u$.

Remark 3.19. Let $(X, G)$ be a rectangular $G$-metric space and $d: X \times X \rightarrow[0, \infty)$ be a function defined by $G(x, y, y)=d(x, y)$ with $\phi(t)=t$ and $\gamma(2 \delta G(x, T x, T x))=0$, then Theorem 3.18 reduces to Banach contraction principle in rectangular-metric space.

Clearly, Remark 3.14 can be applied in the case Remark 3.16.

Example 3.20. Let $X=\mathbb{R}$ endowed with the a generalized rectangular metric $G(x, y, z)=|x-y|+|x-z|+|y-z|$ for all $x, y, z \in X$. Define the mapping $T: X \rightarrow X$ by 


$$
T(x)= \begin{cases}\frac{4}{x}, & \text { if } \quad x>2, \\ 2 x, & \text { if } x \in(0,2], \\ 0, & \text { otherwise. }\end{cases}
$$

At first, it is observed that Banach contraction principle in $G$-metric space introduced by Mustafa and Sims [10] cannot be applied in this case too because

$$
\begin{aligned}
G(T 3, T 1, T 4) & =G\left(\frac{4}{3}, 2,1\right) \\
& =\left|\frac{4}{3}-2\right|+\left|\frac{4}{3}-1\right|+|2-1| \\
& =2 \\
& >k G(3,1,4) \\
& >k(|3-1|+|3-4|+|1-4|) \\
& >6 k=6 \times \frac{1}{8}=\frac{3}{4}, \frac{1}{8} \in[0,1) .
\end{aligned}
$$

But Theorem 3.15 can be applied in this case because

$$
\begin{aligned}
G(T 3, T 1, T 4)= & G\left(\frac{4}{3}, 2,1\right) \\
= & \left|\frac{4}{3}-2\right|+\left|\frac{4}{3}-1\right|+|2-1| \\
= & 2 \\
\leq & \delta G(3,1,4)+2 \delta G\left(\frac{4}{3}, 1,4\right) \\
\leq & \delta\left[G(3,1,4)+2 G\left(\frac{4}{3}, 1,4\right)\right] \\
\leq & \delta[(|3-1|+|3-4|+|1-4|) \\
& \left.+2\left(\left|\frac{4}{3}-1\right|+\left|\frac{4}{3}-4\right|+|1-4|\right)\right] \\
\leq & \frac{56}{3} \times \delta .
\end{aligned}
$$


Since $\delta=\max \left\{a, \frac{b}{1-b}, \frac{c}{1-c}\right\}$. Theorem 3.18 can be applied in this case too because

$$
\begin{aligned}
G(T 3, T 1, T 4)= & G\left(\frac{4}{3}, 2,1\right) \\
= & \left|\frac{4}{3}-2\right|+\left|\frac{4}{3}-1\right|+|2-1| \\
= & 2 \\
\leq & 0.5 \times \delta G(3,1,4)+0.5 \times \delta G\left(3, \frac{4}{3}, \frac{4}{3}\right) \\
\leq & 0.5 \times \delta\left[G(3,1,4)+G\left(3, \frac{4}{3}, \frac{4}{3}\right)\right] \\
\leq & 0.5 \times \delta[(|3-1|+|3-4|+|1-4|) \\
& \left.+\left|3-\frac{4}{3}\right|+\left|3-\frac{4}{3}\right|+\left|\frac{4}{3}-\frac{4}{3}\right|\right] \\
\leq & \frac{79}{18} \times \delta .
\end{aligned}
$$

Since $\delta=\max \left\{a, \frac{b}{1-b}, \frac{c}{1-c}\right\}$.

\section{Concluding Remarks}

In this paper, we present the notion of generalized rectangular metric space which extends a rectangular metric space. We also use an example to show that the newly introduced generalized rectangular metric space is better than rectangular metric space and different from $G$-metric spaces. Some fixed point theorems are stated and proved in this new space.

\section{Acknowledgement}

The authors are grateful to the anonymous referee whose comments improved the original version of this manuscript. 


\section{References}

[1] O. K. Adewale, J. O. Olaleru, H. Olaoluwa and H. Akewe, Fixed point theorems on a $\gamma$-generalized quasi-metric spaces, Creative Mathematics and Informatics 28(2) (2019), 135-142.

[2] O. K. Adewale, J. O. Olaleru and H. Akewe, On quasiconvex metric spaces, Advanced Fixed Point Theory 10 (2020), 1-11.

DOI: https://doi.org/10.28919/afpt/4770

[3] O. K. Adewale, J. O. Olaleru and H. Akewe, Fixed point theorems on a quaternionvalued $G$-metric spaces, Communications in Nonlinear Analysis 7(1) (2019), 73-81.

[4] O. K. Adewale and K. Osawaru, G-cone metric spaces over Banach algebras and some fixed point results, International Journal of Mathematical Sciences and Optimization: Theory and Applications 2 (2019), 546-557.

[5] O. K. Adewale, J. C. Umudu and A. A. Mogbademu, Fixed point theorems on $A_{p}$-metric spaces, International Journal of Mathematical Sciences and Optimization: Theory and Applications 1 (2020), 657-668.

[6] A. Branciari, A fixed point theorem of Banach-Caccippoli type on a class of generalized metric spaces, Publicationes Mathematicae 57(1-2) (2000), 31-37.

[7] B. C. Dhage, Generalized metric space and mapping with fixed point, Bulletin of the Calcutta Mathematical Society 84 (1992), 329-336.

[8] M. Frechet, Sur quelques points du calcul fonctionnel, Rendiconti del Circolo Matematico di Palermo 22 (1906), 1-72.

[9] S. Gahler, 2-Metrische Raume und ihre topologische Struktur, Mathematishe Nachrichten 26(1-4) (1963), 115-148.

DOI: https://doi.org/10.1002/mana.19630260109

[10] Z. Mustafa and B. Sims, A new approach to generalized metric spaces, Journal of Nonlinear and Convex Analysis 7(2) (2006), 289-297.

[11] J. O. Olaleru, Common fixed points of three self-mappings in cone metric spaces, Applied Mathematics E-Note 11 (2011), 41-49.

[12] J. O. Olaleru and B. Samet, Some fixed point theorems in cone rectangular metric spaces, Journal of the Nigerian Mathematical Society 33(1-3) (2014), 145-158. 